\title{
Post-infectious encephalomyelitis associated with Mycoplasma pneumoniae and Legionella pneumophila infection
}

\author{
Philippa J. Easterbrook ${ }^{1}$ and Edward G. Smyth ${ }^{2}$
}

Departments of ${ }^{1}$ Medicine and ${ }^{2}$ Medical Microbiology, St George's Hospital, London SW17, UK

\begin{abstract}
Summary: A case of severe acute encephalomyelitis associated with a serological diagnosis of both Mycoplasma pneumoniae and Legionella pneumophila infection is reported. Serological co-positivity between these two pathogens has been reported previously, and has, in general, been attributed to cross-reactivity. This is the first documented case of co-infection using more sensitive and specific serological techniques. The potential significance of these findings is discussed in the context of the considerable problems in the interpretation of serological data.
\end{abstract}

\section{Introduction}

The simultaneous or sequential occurrence of infection with 2 or more pathogens is well recognized, ${ }^{1}$ although how such mixed infections influence clinical presentation and outcome remains poorly understood. Both Mycoplasma pneumoniae and Legionella pneumophila are important causes of pneumonia in man, and there are many reports of co-infection between these and other pathogens. ${ }^{2-4} \mathrm{~A}$ high degree of serological co-positivity between $M$. pneumonia and $L$. pneumophila has also been reported in the United States, ${ }^{5-8}$ although these findings have not been confirmed in this country, using an indirect fluorescent antibody test and a more specific formalin inactivated yolk sac antigen for the detection of L. pneumophila. ${ }^{9-11}$ It has been suggested that this observed co-positivity may be due to serological cross-reactivity through the use of relatively non-specific preparations of legionella antigen, or may reflect true geographical differences in the patterns of infection with these organisms between the 2 countries. Overall, the consensus to date has been that true dual infection with these pathogens is rare. There is only one previous report of definite dual infection in the literature, and in this case the diagnosis of legionella infection was established by direct immunofluorescence and not serologically. ${ }^{6}$ We report a further case of probable co-infection with $M$. pneumoniae and L. pneumophila, diagnosed using the more specific techniques employed in the UK.

Correspondence: P.J. Easterbrook, M.D., M.R.C.P., St Stephen's Hospital, 369 Fulham Road, London SW10 9TH, UK

Accepted: 18 April 1991

\section{Case report}

A previously healthy 19 year old male was admitted to St George's Hospital, London in a deep coma. During the week prior to admission he had de $\delta$ veloped a sore throat and productive cough, an had become increasingly apathetic and drowsy. Ot] examination, the patient was comatose with temperature of $39.5^{\circ} \mathrm{C}$, blood pressure of $150 / 90$ $\mathrm{mmHg}$ and a pulse rate of $130 / \mathrm{min}$. There was evidence of meningism but no focal neurological signs. Physical examination was otherwise normal. Initial investigations showed an elevated white blood cell count of $17 \times 10^{9} / 1$ (81\% neutrophils), but electrolytes and liver function tests were normal. Chest X-ray revealed patchy infiltrates at the left lung base. Arterial blood gases on air were: $\mathrm{PO}_{2}$ $5 \mathrm{kPa}, \mathrm{PCO}_{2} 4 \mathrm{kPa}$, and $\mathrm{pH} 7.41$. Examination of cerebrospinal fluid (CSF) revealed an increased pressure of $27 \mathrm{~cm}, 3$ red cells $\times 10^{6} / 1,18$ white cells $\times 10^{6} / 1(14 \%$ neutrophils $)$, protein $0.70 \mathrm{~g} / 1$ and a normal glucose. No acid fast bacilli were present on sputum examination and culture yielded upper respiratory tract flora only. Repeated culture of blood and CSF for bacteria (including Mycobacterium sp.) and viruses yielded no growth. Electroencephalogram (EEG) showed diffuse slowing compatible with brain stem encephalitis. A noncontrast computerized tomographic (CT) scan was normal.

A presumptive diagnosis of encephalitis associated with an atypical pneumonia was made, but the possibility of both Herpes simplex encephalitis and a partially treated bacterial meningitis was considered. The patient was therefore treated with a combination of intravenous benzylpenicillin $2.4 \mathrm{~g}$ 
4 hourly, chlorampenicol $500 \mathrm{mg} 6$ hourly and acyclovir $500 \mathrm{mg} 8$ hourly. The following day, he deteriorated and required elective tracheostomy and mechanical ventilation. Neurological examination now revealed a complete quadriparesis below $\mathrm{C} 2$, with loss of all tendon reflexes, and no response to painful stimuli. Over the next few days, his pupillary reflexes became unequal, and the gag reflex was lost bilaterally, although his upper brain stem reflexes were preserved. A repeat lumbar puncture on the 6th day following admission revealed 28 red blood cells $\times 10^{6} / 1,42$ white blood cells $\times 10^{6} / 1 \quad(50 \%$ lymphocytes $)$, a protein of $0.95 \mathrm{~g} / 1$ and a sterile CSF culture. Insufficient CSF was obtained to measure CSF immunoglobulins. $\mathrm{He}$ was treated with dexamethasone, and the antibiotics were changed to intravenous cefotaxime $2 \mathrm{~g} \mathrm{8}$-hourly and erythromycin $500 \mathrm{mg} \mathrm{6}$-hourly. Acyclovir was continued.

Two days later he had a massive haematemesis. Gastroscopy identified an actively bleeding anterior duodenal ulcer and he underwent a partial gastrectomy. Twenty-four units of blood were transfused during this period. His subsequent clinical course was characterized by a low grade febrile illness associated with intermittent lobar collapse and liver dysfunction.

Serology was performed in the Department of Microbiology at St George's Hospital on acute serum taken in the second week of his illness and on serial convalescent sera. Aliquots of these specimens were also sent to 3 other laboratories for confirmation. A summary of their findings is presented in Table I. The detection of positive cold agglutinins, and $M$. pneumoniae specific igM, by both enzyme linked immuno-absorbent assay (ELISA) and indirect haemagglutination assay (IHA), together with complement fixing (CF) antibody titre of 256 were considered diagnostic of recent $M$. pneumoniae infection. Mycoplasma IgM and a CF titre of 16 were also detected in the CSF during the 3rd week following admission. Further aliquots of serum were sent to the Mycoplasma Reference Laboratory which found a positive mycoplasma IgM using indirect immuno-fluorescence (IFA), suggestive of recent infection. The sera were also tested retrospectively by 2 laboratories for the presence of antibodies to L. pneumophila. A legionella yolk sac antigen serogroup 1 was used in the indirect fluorescent antibody test (IFAT), and the rapid micro-agglutination antibody test (RMAT) antigen was prepared from a formalin-inactivated suspension of bacteria (serogroup 1) grown on solid media. Both antigens were supplied by the Central Public Health Laboratory (CPHL). By convention, a serological diagnosis of L. pneumophila infection is based on either a 4-fold rise in the IFAT titre to $\geqslant 64$ (or a single titre of $\geqslant 128$ ), or a 4-fold rise in RMAT titre of $\geqslant 16$ (or a single titre of $\geqslant 32$ ). ${ }^{12}$ Legionella infection in this case was inferred from the presence of a significant titre rise in both tests and in both laboratories during the 4th week of admission and from a CSF titre of 16. Serum aliquots were also analysed by the Legionella Reference Laboratory, which found non-diagnostic titres. However, on review it was apparent that the vials had been repeatedly frozen and thawed, and not vortexed prior to dispatch to the Reference Laboratory. In view of this sampling error, these results were omitted from the table.

Routine serological tests for antibodies to other agents were all negative, other than a late rise in Epstein-Barr virus (EBV) IgG and IgA and antiEpstein-Barr nucleic antigen (anti-EBNA) titres, associated with an atypical lymphocytosis, during the 11 th week of admission. This was considered to represent a transfusion-acquired EBV infection following the massive transfusion in the 3rd week of admission.

Over the course of the next 6 weeks there was a slow improvement in his neurological state. By the 8th day he was alert and orientated, and able to blink in response to commands; by the 14th day he could lateralize his gaze to command and after a month, some movement of his mouth and tongue appeared. Six weeks following admission, a magnetic resonance imaging scan demonstrated the presence of multifocal cortical (mainly white matter) disease. An EEG was within normal limits and an electromyelogram showed severe widespread denervation in all limbs, compatible with anterior horn cell and motor root involvement, with preserved sensory action potentials.

There has been no further evidence of neurological recovery: he remains quadriplegic, able to move his eyes, and to mouth words but not to speak or swallow.

\section{Discussion}

The difficulties in establishing a serological diagnosis of infection are well recognized. Serology is an indirect method of detecting the presence of an infectious agent, with relatively poor sensitivity and specificity. Furthermore, in the absence of international gold standards, the serological techniques may vary, resulting in considerable problems of quality control. However, at least for the serodiagnosis of $M$. pneumoniae ${ }^{13}$ L. pneumophila ${ }^{12}$ and Epstein-Barr virus ${ }^{14}$ infections, there are well-established guidelines. Our patient fulfilled the necessary serological criteria for recent infection with all 3 agents. The diagnosis of mycoplasma infection was suggested by 3 laboratories, each using different techniques and $L$. pneumophila antibody was detected by 2 laboratories. The discrepant findings of the Legionella Reference 
Table I Serological titres for Legionella pneumophila, Mycoplasma pneumoniae and Epstein-Barr virus in different laboratories

\begin{tabular}{|c|c|c|c|c|c|}
\hline \multirow[b]{2}{*}{ Laboratory } & \multicolumn{5}{|c|}{ Week of illness* } \\
\hline & 2 & 3 & 4 & 7 & 11 \\
\hline \multicolumn{6}{|l|}{ M. pneumoniae } \\
\hline Cold agglutinins $\dagger$ & Positive & e - & - & - & - \\
\hline CFT $\dagger$ & 256 & 128 & 64 & 32 & - \\
\hline IgG IFA§ & $<32$ & - & $<32$ & 32 & - \\
\hline IgM IFA§ & $<8$ & - & $<8$ & 8 & - \\
\hline IgM ELISA $\dagger$ & Positive & e - & Positive & - & - \\
\hline IgM IHA & - & 80 & - & 10 & - \\
\hline $\mathrm{CSF}$ CFT $\dagger$ & - & 16 & - & - & - \\
\hline CSF IgM ELISA $\dagger$ & - & Positive & e - & - & - \\
\hline \multicolumn{6}{|l|}{ L. pneumophila } \\
\hline RMAT $\dagger$ & $<8$ & $<8$ & 16 & $<8$ & - \\
\hline (Serogroup 1) $\ddagger$ & $<8$ & - & 32 & - & - \\
\hline & $<8$ & 8 & - & 16 & - \\
\hline IFAT $†$ & $<16$ & 64 & 64 & 64 & - \\
\hline (Serogroup 1) $\ddagger$ & $<16$ & - & $>512$ & - & - \\
\hline \multicolumn{6}{|l|}{ Epstein-Barr virus } \\
\hline IgG to viral capsid Ag. $\dagger$ & $<8$ & 16 & - & - & 256 \\
\hline IgA to viral capsid Ag. $\dagger$ & $<8$ & $<8$ & - & - & 16 \\
\hline Anti-EBNA $\dagger$ & $<4$ & 8 & - & - & 16 \\
\hline
\end{tabular}

*Transfusion of 24 units of blood occurred between the 3rd and 4th weeks of illness; †Department of Medical Microbiology, St George's Hospital; $\ddagger$ Department of Virology, Brompton Hospital; §Mycoplasma Reference Laboratory, CPHL; -Clinical Microbiology and Public Health Laboratory, Addenbrooke's Hospital, Cambridge. CFT = complement fixation test; ELISA = enzyme-linked immunoabsorbent assay; IFA = indirect immuno-fluorescence assay; IHA = indirect haemagglutination assay; $\mathrm{RMAT}=$ rapid micro-agglutination antibody test; IFAT = indirect fluorescent antibody test; anti-EBNA = anti-Epstein-Barr nucleic antigen.

Laboratory were attributed to a sampling error, as discussed above.

There are several possible interpretations of these findings. Firstly, both $L$. pneumophila and $M$. pneumoniae may have cross-reacted with a single antibody in the patient's serum, generating a false positive reaction. This phenomenon probably accounts for the majority of cases of co-infection reported previously, ${ }^{5-8}$ because of the use of a cross-reactive heat or ether-killed L. pneumophila antigen grown on solid media. ${ }^{9-11}$ However, the use, as in this case, of a formalin-killed yolk sac legionella antigen has largely overcome this problem. Subsequent studies have shown that crossreactions are uncommon using this antigen..$^{9-11}$

Another interpretation is that the rise in antibody titres was spurious, due to non-specific polyclonal B cell activation. Biberfeld and others have demonstrated that $M$. pneumoniae is a B-lymphocyte activator capable of inducing non-specific activation of memory B cells in vivo. ${ }^{15-17}$ Such an anamnestic response to $M$. pneumoniae has been reported with EBV and one possible case with legionella infection. ${ }^{18}$
Thirdly and more likely is that true co-infection $\overline{\overrightarrow{0}}$ with $M$. pneumoniae and $L$. pneumophila occurred, followed by an EBV infection. It is difficult to establish the precise timing of exposure to the two agents, since the incubation period and mean interval to seroconversion vary widely for both infections. Seroconversion to legionella, for example, often occurs several weeks after the onset of the disease. ${ }^{19}$ There was an early peak during the $\frac{O}{3}$ second week in mycoplasma IgG and IgM antibody titres, and the IgM titres remained elevated into the fourth week, which was also the time of the $\frac{7}{0}$ rise in antibody titres to $L$. pneumophila. $M$. pneumoniae is also known to suppress cellular $N$ immunity, ${ }^{17}$ and immunocompromised patients are $N$ predisposed to legionella infection. This, together $\mathrm{N}^{\circ}$ with the serology, suggest that $M$. pneumoniae was $\omega$ the primary pathogen with a secondary L. pneumophila infection. The timing of the rise in EBV $\stackrel{O}{\subset}$ antibody titres, shortly after the massive blood $\mathbb{\Phi}$ transfusion and the subsequent rise in $\mathrm{IgG}$ and anti-EBNA noted 9 weeks later, is strongly suggestive of a transfusion-acquired infection. An $\underset{\mathbb{D}}{ }$ alternative explanation is that this was simply a $\frac{?}{\mathbb{D}}$ 
reactivation of a latent EBV infection by $M$. pneumoniae. However, this interpretation is precluded by the initial negative anti-EBNA titre, which would be expected to remain positive indefinitely following a primary infection. ${ }^{14}$

The overall clinical presentation and course provide no additional insight as to the likely contribution and time relationship of the two infections. L. pneumophila infection resembles $M$. pneumoniae, particularly with respect to clinical manifestations and neurological complications. ${ }^{20-22}$ However, it is possible that the subsequent febrile illness associated with liver dysfunction, initially assumed to be part of a drug reaction, was in fact a manifestation of $L$. pneumophila infection or possibly even early EBV infection. The absence of a history of recent travel, time spent in an airconditioned building, legionella antibodies in the sera of other patients on the intensive care unit, or of any other recent local cases of Legionnaire's disease, suggest that this was probably a sporadic case of L. pneumophila.

The pathogenesis of the CNS disease with $M$. pneumoniae is unknown. Four hypotheses have been proposed and include direct invasion of the central nervous system by the organism, production of a neurotoxin, autoimmune mechanisms, or vascular damage. ${ }^{22}$ Confirmatory isolation of the organism in the CSF was not possible in this case. However, the presence of $M$. pneumoniae IgM and $\mathrm{CF}$ antibody in the CSF lends some support to the hypothesis of direct central nervous system invasion, although this may simply reflect passage of serum titres across a damaged blood-brain barrier. L. pneumophila is also a recognized infectious cause of neurological damage. While it is impossi- ble to delineate the precise contribution of either agent to the clinical picture, it is conceivable that the two organisms acted synergistically in the pathogenesis of the neurological disease, as has been postulated with cytomegalovirus and the human immunodeficiency virus (HIV). ${ }^{23}$

This case serves to highlight some of the problems in the serological diagnosis of legionella and mycoplasma infections. In recent years, improved culture techniques and diagnostic methods of antigen detection, such as indirect immunofluorescence with monoclonal antibodies and enzyme linked immunoassay, ${ }^{24}$ have been developed. In addition, cDNA probes ${ }^{25}$ and the polymerase chain reaction have been used to detect mycoplasma ribosomal RNA and DNA, respectively. Although these techniques are currently available in only a few specialized laboratories, they offer the potential for the future to characterize more precisely the epidemiology of mycoplasma and legionella infection and further cases of dual infection. Finally, it would seem appropriate in cases of acute meningoencephalitis or related central nervous system pathology where no cause has been established, to search also for evidence of both $M$. pneumoniae and $L$. pneumophila infection even in the absence of pneumonia, and to consider the early empirical use of intravenous erythromycin.

\section{Acknowledgements}

We are grateful to Dr R. Fallon, Ruchill Hospital, Glasgow and Dr N. Leigh at St George's Hospital for their comments on this case, and to Drs T. Harrison, A. Taylor and R. Leech at the Central Public Health Laboratory for assistance in the interpretation of the serological results.

\section{References}

1. Loosli, C. Influenza and the interaction of viruses and bacteria in respiratory infections. Medicine 1973, 52: 384.

2. Fekety, R.F., Caldwell, J., Gump, D. et al. Bacteria, viruses and mycoplasmas in acute pneumonia in adults. Am Rev Respir Dis 1971, 104: 499-507.

3. Helms, C.M., Viner, J.P., Renner, E.D., Chiu, L.C. \& Weisenburger, D.D. Legionnaires' disease among pneumonia in Iowa (1972-1978). II. Epidemiologic and clinical features of 30 sporadic cases of $\mathrm{L}$. pneumophila infection. Am J Med Sci 1981, 281: 3-13.

4. Kirby, B.D., Synder, T.M., Meyer, R.D. \& Feingold, S.M. Legionnaire's disease: clinical features of 20 cases. Ann Intern Med 1978, 89: 297-309.

5. Renner, E.D. \& Helms, C.M. Seroactivity of Mycoplasma pneumoniae and Legionella pneumophila: lack of a statistically significant relationship. J Clin Microbiol 1981, 13: 1096-1098.

6. Oldenburger, D., Carson, J., Gundlach, W.J., Ghaly, F.I. \& Wright, W.H. Legionnaire's disease. Association with Mycoplasma pneumoniae and disseminated intravascular coagulation. JAMA 1979, 241: 1269-1270.

7. Renner, E.D., Helms, C.M. \& Hierhoizer, W.T. Legionnaire's disease in pneumonia patients in Iowa. A retrospective seroepidemiologic study, 1972-1977. Ann Intern Med 1979, 90: 603-606.
8. Grady, G.F. \& Gilfillan, R.F. Relation of Mycoplasma pneumoniae seroreactivity, immunosuppression and chronic disease to Legionnaire's disease. Ann Intern Med 1979, 90: 607-610.

9. Taylor, A.G., Harrison, T.G., Andrews, B.E. \& Sillis, M. Serological differentiation of Legionnaire's disease and Mycoplasma pneumoniae. Lancet 1980, i: 764-765.

10. Fleurette, J., Bornstein, N., Aymard, M. \& Bosshard, S. Serological differentiation of Legionnaire's disease and Mycoplasma pneumoniae and Chlamydia psittaci pneumonia. J Infect 1981, 3: 88-89.

11. Holliday, M.G. Legionnaire's disease: diagnosis and differentiation from Mycoplasma pneumoniae pneumonia by counter current electrophoresis. Med Lab Sci 1981, 38: 291-292.

12. Harrison, T.G., Dournon, F. \& Taylor, A.G. Evaluation of sensitivity of two serological tests diagnosing pneumonia caused by Legionella pneumophila serogroup 1. J Clin Path 1987, 40: 77-82.

13. Wreghitt, T.G. \& Sillis, M. A U capture Elisa for detecting Mycoplasma pneumoniae IgM: comparison with indirect immunofluorescence and indirect Elisa. J Hyg Camb 1985, 94: $217-227$. 
14. Henle, W., Henle, G.E. \& Horwitz, C.A. Epstein-Barr virus specific diagnostic tests in infectious mononucleosis. Hum Pathol 1974, 5: 551-565.

15. Biberfeld, G. Activation of human lymphocyte subpopulations by Mycoplasma pneumoniae. Scand J Immunol 1977, 6: $1145-1150$.

16. Biberfeld, G., Arneborn, P., Forsgren, M., Von-Steding, K.L.O. \& Rlomquist, S. Non specific polyclonal antibody response induced by Mycoplasma pneumoniae. Yale $J$ Biol Med 1983, 5: 639-642.

17. Biberfeld, G. \& Sterner, G. Effect of Mycoplasma pneumoniae infection on cell mediated immunity. Infection 1976, 4 (Suppl 1): $17-20$.

18. Littrup, P., Madsen, J.K. \& Lind, K. Aortic valve endocarditis associated with Legionella infection after Mycoplasma pneumoniae. Br Heart J 1987, 58: 293-295.

19. Mandell, G.L., Douglas, R.G. \& Bennett, J.E. In: Principles and Practice of Infectious Diseases, 2nd ed. Wiley Medical Publications, Chichester 1985.

20. Hodges, G.R. \& Fars, R.J. Central nervous system disease associated with Mycoplasma pneumoniae infection. Arch Intern Med 1972, 130: 277-282.
21. Johnston, J.O., Raff, M.J. \& Van Arrdall, J.A. Neurologic manifestations of Legionnaires' disease. Medicine 1984, 63: 5 .

22. Decaux, G., Szyper, M., Ectors, M., Cornil, A. \& Franken, L. Central nervous system complications of Mycoplasma pneumoniae. J Neurol Neurosurg Psychiat 1980, 43: 883-887.

23. Dix, R.D., Bredeson, M.D., Erlich, K.S. \& Mills, J. Recovery of herpes viruses from cerebrospinal fluid of immunodeficient homosexual men. Ann Neurol 1985, 18: 611-614.

24. Kok, T.W., Varkanis, G., Marmion, B.P., Martin, J. \& Esterman, A. Laboratory diagnosis of Mycoplasma pneumoniae infection. 1. Direct detection of antigen in respiratory exudates by enzyme immunoassay. Epidemiol Infect 1988, 101: $669-684$.

25. Harris, R., Marmion, B.P., Varkanis, G., Kok, T., Lunn, B. \& Martin, J. Laboratory diagnosis of Mycoplasma pneumoniae infection. 2. Comparison of methods for the direct detection of specific antigen or nucleic acid sequences in respiratory exudates. Epidemiol Infect 1988, 101: 685-694. 